\title{
Recognition of Bragg Wavelength Disturbed by Time Delay of Fiber Length in Prepositive Tunable Filter
}

\author{
Chuan Li, Xiaoyong Chao, Yingna Li, Tao Xie, Zhengang Zhao, Xin Xiong \\ Faculty of Information Engineering and Automation, Kunming University of Science and Technology, Kunming City, China \\ Email: lichuankmust@163.com
}

Received 2013

\begin{abstract}
The wavelength shift in fiber Bragg grating does not depend directly on the total light levels, losses in the connecting fibers and couplers, or source power. However, if the tunable Fabry-Perot filter is place on the end of incident fiber, the detected time delay of modulation light is occurred due to the unmatch between the scanning time and light transmission time in the transmission fiber. Consequently, the detected peak wavelength shifts with the length of transmission fiber. Thus, the peak wavelength shift effect of Bragg reflective light transmitted in fiber with different fiber length can be obvious in the demodulator with a prepositive tunable Fabry-Perot filter. The experiment indicates the shift rates of $0.109-0.126 \mathrm{~nm} / \mathrm{km}$ increase approximately linearly with the original peak wavelength of $1532.917-1560.300 \mathrm{~nm}$ at the fiber length of $0-6 \mathrm{~km}$. To certify the consistency of measurement data, the criterion correction is introduced. By using the differential method of two fiber Bragg gratings with an optical path, the differential worth is compensated from the disturbance modulated by the time delay of fiber length.
\end{abstract}

Keywords: Optic Fiber; Fiber Length; Bragg Wavelength; Fabry-Perot Filter; Time Delay

\section{Introduction}

Fiber photosensitivity was first observed in germaniumdoped silica fiber in experiments performed by K. Hill and coworkers at the communication research center in Canada in 1978 [1,2]. Since then, the fiber Bragg gratings represent a key element in the established and emerging fields of optical communications and optical fiber sensing. The principal advantage is that the measurand information is wavelength-encoded (an absolute quantity, or a state value), thereby making the sensor self-referencing, rendering it independent of fluctuating light levels and the system immune to source power and connector losses [3-5]. In the sensing system of fiber Bragg grating, the precision of shift of peak wavelength $\Delta \lambda_{B}$ should be superior to $0.001 \mathrm{~nm}$ for proving the measurement precision of $0.1^{\circ} \mathrm{C}$ or $1 \mu \varepsilon$. Thus, the measurement precision $\Delta \lambda_{B}$ defined the measurement precision of whole system. However, the intensity variations in the light source, the bending on lead/in-out fibers, and a tunable filter can cause signal deviations when a tunable filter scans the Bragg grating sensors [6-8]. H. G. Limberger etc. found a reflectivity of $94 \%$ and a bandwidth of $1.7 \mathrm{~nm}$ in communication fiber with three sidelobes [6]. V. Gaillarda etc. research the optical spectrum feature in the fiber of low coherence interferometer and fiber Bragg grating [7]. Y. L. Lo etc. found that the in- tensity variations from the macrobending of the lead-in/ out fibres during high frequency disturbances can cause deviations of the signal when a tunable filter scans the Bragg wavelength [8].

\section{Peak Wavelength Shift with the Fiber Length in a Prepositive Tunable Filter}

The fiber Bragg grating is a permanent perlodic modulation of the refractive index along a given length of optical fiber. Due to the coupling between the forward and backward propagating modes, the specific wavelength light depending on the modulation period of the refractive index is reflected at the location of the fiber Bragg grating. The other wavelength lights are transmitted through the fiber Bragg grating. The incident light is reflected when its peak wavelength is equal to the Bragg wavelength [1-5]:

$$
\lambda_{\max }=\lambda_{\text {Bragg }}=2 n_{\text {eff }} \Lambda
$$

where, $n_{\text {eff }}$ is the effective refractive index of reverse couple mode, $\Lambda$ is the period of grating. The most important property of FBG is that it will reflect the incident light with particularly predetermined wavelengths, while passing all the other wavelengths of light at the same time. As the wavelength of the reflected light varies with the strain, temperature and the other environmental fac- 
tors, detection of the wavelength will yield information about these quantities. Figure $\mathbf{1}$ is the schematic diagram of fiber Bragg grating sensing modulation system, whereinto, (a) is the reflection-type measurement, and (b) is the transmission-type measurement. In the modulation system, the light sources can be broadband light, tuning light, pulse light, and laser etc., the connectors are coupler and circulator, the sensing gratings are fiber Bragg grating and long period fiber grating, and the photoelectric detector is used [5].

In the modulation process, the incident light directly accessed the sensing grating through the transmission optical path or the optical coupler. Under the static, quasistatic, and time varying action of external filed, such as: strain filed, temperature filed ect., the incident light is modulated; subsequently, the reflected (or transmitted) modulated light is detected by the photoelectric detector. A very important character of fiber Bragg grating is that the peak wavelength $\lambda_{B}$ of reflected light will change when there is a change in strain, temperature and other environment factor. The reason is that change of the strain of the grating or its environmental temperature can result in change in the efficient refractive index of the core $n_{\text {eff }}$ as well as the period of index modulation $\Lambda$. Therefore, the the shift of Bragg wavelength $\Delta \lambda_{B}$ can be expressed as:

$$
\Delta \lambda_{\text {Bragg }}=2 n_{\text {eff }} \Delta \Lambda+2 \Delta n_{\text {eff }} \Lambda
$$

where, $\Delta \Lambda$ is the elastic deformation of fiber; $\Delta n_{\text {eff }}$ is the elasto-optical effect of fiber. Eq. (2) indicate that the effective measurement of $\Delta \lambda_{B}$ determine the measurement precision of the whole system.

The peak wavelength shift effect of Bragg reflective light transmitted in fiber is observed, as shown in Figure 2. In this experiment, the fiber is the G.652 standard communication monomode fiber, and the fiber Bragg grating is inscribed in the Hydrogen-loaded G.652 fiber.

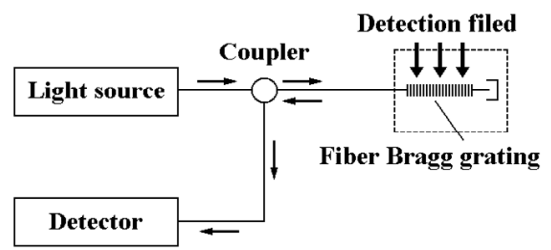

(a) Reflection-type

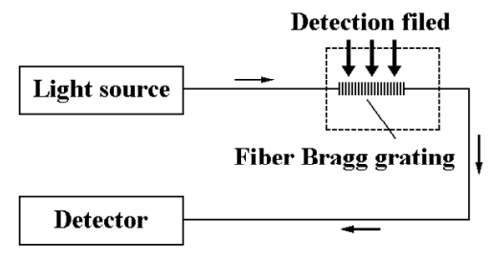

(b) Transmission-Type

Figure 1. The schematic diagram of fiber Bragg grating sensing demodulation system.
In Figure 2, the incident broadband light source is modulated to the narrowband light by the tunable FabryPerot (TFP) filter. The filter is characterized by bandpass resonances of Lorentzian lineshape and bandwidth of typically $0.3 \mathrm{~nm}$, with a wide operating range of $40 \mathrm{~nm}$, depending on the spacing between the mirrors of FabryPerot etalon. Electrical control of this mirror spacing via piezoelectric stacks allows for tuning the passband wavelength. In operation, the passband light is injected to each fiber Bragg grating through two couplers, a circulator. The return passband light modulated by the grating is detected by the detector through the circulator. As the filter is tuned, the passband scans over the return signal from the grating, and the wavelength can be determined and recorded from the matching peak wavelength of reflective light.

In this scanning optical filter, the wavelength range is $1525-1565 \mathrm{~nm}$, the wavelength resolution is $0.001 \mathrm{~nm}$, and the wavelength repetition is $0.01 \mathrm{~nm}$. In this experiment, the tunable Fabry-Perot filter discovers that the peak wavelength is shifted with the variation of fiber length, as shown in Figure 3.

In Figure 3, the original peak wavelengths of grating are $\lambda_{\max }(l=0)=1532.917 \mathrm{~nm}, 1537.091 \mathrm{~nm}, 139.899 \mathrm{~nm}$, $1540.568 \mathrm{~nm}, 1545.063 \mathrm{~nm}, 1550.444 \mathrm{~nm}, 1555.573 \mathrm{~nm}$, and $1560.300 \mathrm{~nm}$, and the widths of gratings are $\Delta \lambda=0.2$ $\mathrm{nm}$. As the fiber length is $l=6 \mathrm{~km}$, the peak wavelength shifts are $0.654-0.759 \mathrm{~nm}$. By using fitting, the peak wavelength shift of Bragg reflective light $\lambda_{\max }(l)-\lambda_{\max }(0)$ is related to the fiber length $l$ :

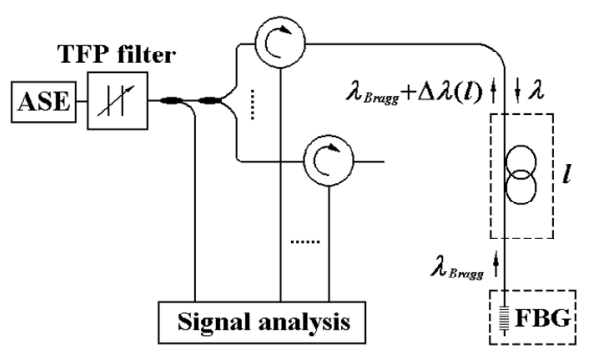

Figure 2. The schematic diagram of the fiber Bragg grating measurement system demodulated via a prepositive tunable Fabry-Perot passband filter.

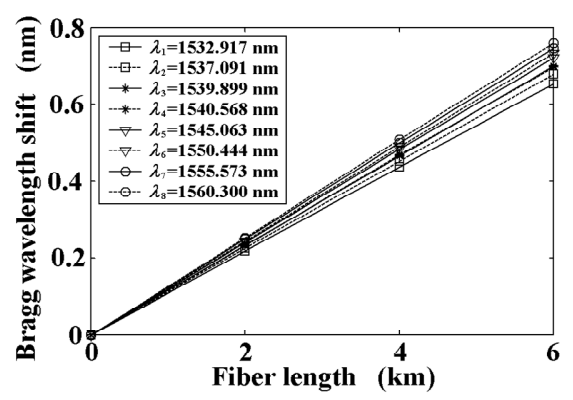

Figure 3. The peak wavelength shift of Bragg reflective light related to the fiber length. 


$$
\begin{aligned}
& \lambda_{\max }(l)-\lambda_{\max }(0) \\
= & \left\{0.109+\left[\lambda_{\max }(0)-1532.917\right] \times 6.823 \times 10^{-4}\right\} l
\end{aligned}
$$

where, the standard error between the fitting results and the experimental results is $0.009 \mathrm{~nm}$, the shift rates of the Bragg wavelength to the fiber length are $0.109-0.126$ $\mathrm{nm} / \mathrm{km}$.

\section{Transmission Component of Bragg Reflective Light}

This wavelength shift effect of Bragg reflective light caused by the fiber length is important to the fiber Bragg grating sensor network. Figure 4 shows the strain experiment based on the cantilever beam of constant bending rigidity $[9,10]$. The bottom of the beam is fixed on the bracket, and the top is hung through the blade, the pothook, and the weight. The cantilever beam is made from the stainless steel material at the size of $l=300.0$ $\mathrm{mm}, h=3.0 \mathrm{~mm}$, and $B=45.9 \mathrm{~mm}$.

In Figure 4, the fiber Bragg gratings mounted on the up and down surfaces of cantilever are separately suffered the strains of $\varepsilon$ and $-\varepsilon$, but is located in the same temperature field $\mathrm{T}$. Thus, the strain $\lambda$ can be expressed as the function of the shifts of Bragg wavelength in the gratings [7-10]:

$$
\varepsilon=\frac{\Delta \lambda_{B}(\varepsilon, \Delta T)-\Delta \lambda_{B}(-\varepsilon, \Delta T)}{2 S_{\varepsilon}}
$$

where, $\mathrm{S}=1.22 \times 10^{-3} \mathrm{~nm} / \mu \varepsilon$ is the strain sensitivity coefficient of grating, $\Delta \lambda_{B}(\varepsilon, \Delta T)$ and $\Delta \lambda_{B}(-\varepsilon, \Delta T)$ are the wavelength shifts of gratings mounted on the up and down surfaces of the cantilever. The strain of the beam caused by the loading can be measured directly by the tunable Fabry-Perot detector (TFP detector), as shown in Figure 5.

In Figure 5, the Bragg wavelengths of gratings mounted on the up and down surface of cantilever are separately subject to wavelength levels of 1550.2 - $1552.2 \mathrm{~nm}$ and 1538.8 - $1540.8 \mathrm{~nm}$. In the loading, the solid and virtual line groups denote the wavelength sensitivities of the gratings mounted on the up and down surface with the

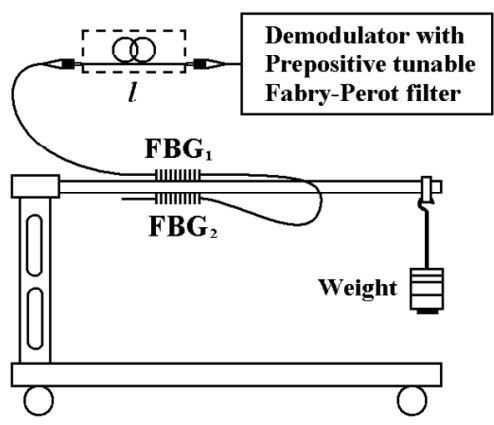

Figure 4. The schematic diagram of loading test.

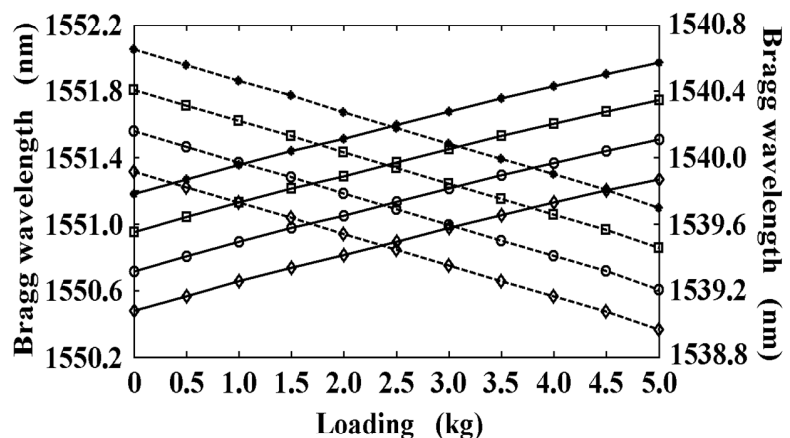

Figure 5. The loading-wavelength variaiton diagram of fiber Bragg gratings mounted on the cantilever with the fiber length of $I=0 \mathrm{~km}, 2 \mathrm{~km}, 4 \mathrm{~km}$, and $6 \mathrm{~km}$.

fiber length of $1=0 \mathrm{~km}, 2 \mathrm{~km}, 4 \mathrm{~km}$, and $6 \mathrm{~km}$. According to Eq. (4), through the least-square algorithm fitting, the strain can be formatted by the weight $\mathrm{g}$ :

$$
\varepsilon=163.1 \mathrm{~g}
$$

where, the strain precision of the gratings is $0.5 \%$ in the strain range of $0-815.6 \mu \varepsilon$. Eq. (5) indicates the Bragg wavelength shift of sensing grating can be obtained by processing these Bragg wavelengths of gratings in the same fiber length, i.e., the Bragg wavelength shift of sensing grating is independent to the Bragg wavelength shift of transmission fiber with different lengths. By using the differential method of two fiber Bragg gratings with an optical path, the differential worth is compensated from the disturbance modulated by the time delay of fiber length.

\section{Conclusions}

In this paper, the shift effect of peak wavelength of Bragg reflective light is produced by changing the length of transmission fiber. The experiment indicates that the peak wavelength shifts of Bragg reflective light are 0.109 - $0.126 \mathrm{~nm} / \mathrm{km}$, which increased with the original peak wavelength of 1532.917 - $1560.300 \mathrm{~nm}$ at the fiber length of $l=0 \mathrm{~km}$. The further sensing experiment indicates that the shift of Bragg wavelength in the prepositive tunable filter includes the sense component of grating and the transmission component of Bragg reflective light. The measured peak wavelength should be corrected when the transmission shift distributes the measurement accuracy. It is noteworthy that the differential worth is compensated from the disturbance modulated by the time delay of fiber length by using the differential method of two fiber Bragg gratings with an optical path.

\section{REFERENCES}

[1] K. O. Hill, Y. Fujii, D. C. Johnson and B.S. Kawasaki, "Photosensitivity in Optical Fiber Waveguides: Application to Reflection Filter Fabrication," Applied Physics 
Letters, Vol. 32, No. 10, 1978, pp. 647-649. doi:10.1063/1.89881

[2] G. Meltz, W. W. Morey, W. H. Glenn and J. D. Farina, "In-Fiber Bragg-Grating Temperature and Strain Sensors, Instrumentation in the Aerospace Industry,” Vol. 34, No.2, 1988, pp. 239-242.

[3] A. D. Kersey, M. A. Davis, H. J. Patrick, M. LeBlanc, K. P. Koo, C. G. Askins, M. A. Putnam and E. Joseph Friebele, "Fiber grating sensors," IEEE Journal of Lightwave Technology, Vol. 15, No. 8, 1997, pp. 1442-1463. doi:10.1109/50.618377

[4] T. Erdogan, "Fiber Grating Spectra," IEEE Journal of Lightwave Technology, Vol. 15, No. 8, 1997, pp. 1277-1294. doi:10.1109/50.618322

[5] C. Li, Y. M. Zhang, Y. G. Zhao and L. J. Li, "Fiber grating: Principles, Techniques and Applications,” Science
Press, Beijing, 2005.

[6] H. G. Limberger, P. Y. Fonjallaz and R. P. Salathe, "Spectral Characterisation of Photoinduced High Efficient Bragg Gratings in Standard Telecommunication Fibres,” Electronics Letters, Vol. 29, No. 1, 1993, pp. 47-49. doi:10.1049/el:19930031

[7] V. Gaillarda, X. Aduriza, N. Daherab, et al., "Local and Spectral Characterization of Optical Fibers and Fiber Bragg Gratings with Low Coherence Interferometry," Fiber and Integrated Optics, Vol. 28, No. 1, 2008, pp. 108-126. doi:10.1080/01468030802272575

[8] Y. L. Lo, J. F. Huang, P. H. Sung and M. D. Yang, "Intensity Effects in Bragg Grating Sensors Scanned by a Tunable Filter," Measurement Science and Technology, Vol. 11, No. 10, 2000, pp. 1456-1462. doi:10.1088/0957-0233/11/10/306 